\title{
Bending Deformation of $\mathrm{C}_{60}$ Nanowhiskers in Solution and Air
}

\author{
Mami Watanabe Non-member (Yokohama City University) \\ Kun'ichi Miyazawa Member (National Institute for Materials Science) \\ Kenichi Kojima Non-member (Yokohama City University) \\ Masaru Tachibana Member (Yokohama City University)
}

Keywords : $\mathrm{C}_{60}$ nanowhiskers, mechanical property, raman spectroscopy

$\mathrm{C}_{60}$ nanowhiskers $\left(\mathrm{C}_{60} \mathrm{NWs}\right)$ have been grown in solution by liquid-liquid interfacial precipitation method. The typical $\mathrm{C}_{60} \mathrm{NWs}$ are less than $0.5 \mu \mathrm{m}$ in diameter and more than $100 \mu \mathrm{m}$ in length. The unique shapes of $\mathrm{C}_{60} \mathrm{NWs}$ are attractive for the applications to nanoscale devices. However, almost all studies on the physical properties have been carried out in air or vacuum.

It is known that the structure of $\mathrm{C}_{60} \mathrm{NWs}$ in air or vacuum is different from original one grown in solution. The $\mathrm{C}_{60} \mathrm{NWs}$ in solution have a hexagonal solvated structure. The solvated structure rapidly changes into a face-centered-cubic (fcc) structure by the drying, i.e. the evaporation of solvent molecules in air or vacuum. Therefore, it is of interest to know what $\mathrm{C}_{60} \mathrm{NWs}$ in solution show physical properties. In this paper, we report the mechanical property of $\mathrm{C}_{60} \mathrm{NWs}$ in solution and air.

The mechanical properties of $\mathrm{C}_{60}$ nanowhiskers in solution and air were investigated by the bending deformation. The bending deformation was carried out by applying wind pressure. The observation of the bending behavior was carried out in-situ by an optical microscope with a charge coupled device.

Fig. 1 shows the successive pictures of the bending behavior of a $\mathrm{C}_{60} \mathrm{NW}$ in solution. The straight $\mathrm{C}_{60} \mathrm{NW}$ before applying wind pressure is indicated by arrow in Fig. 1(a). The $\mathrm{C}_{60} \mathrm{NW}$ was bended with wind pressure and reached the curvature of $290 \mu \mathrm{m}$, as shown in Fig. 1(b). Freeing from the wind pressure, the $\mathrm{C}_{60} \mathrm{NW}$ recovered to original straight shape, as shown in Fig. 1(c). Such behavior of the bending show that $\mathrm{C}_{60} \mathrm{NWs}$ in solution exhibit large elastic deformation.

The bending experiments were also carried out for $\mathrm{C}_{60} \mathrm{NWs}$ in air. The $\mathrm{C}_{60} \mathrm{NW}$ was bended under wind pressure, similar to $\mathrm{C}_{60} \mathrm{NWs}$ in solution. However, the $\mathrm{C}_{60} \mathrm{NW}$ in air was broken just after it was bended with the curvature of only $650 \mu \mathrm{m}$. This indicates that $\mathrm{C}_{60} \mathrm{NWs}$ in air exhibit smaller elastic deformation, i.e. relatively brittle, compared with that in solution. It is obvious that $\mathrm{C}_{60} \mathrm{NWs}$ in solution show abnormal large elastic deformation. This might be due to the solvated structure with weak intermolecular interaction for $\mathrm{C}_{60} \mathrm{NWs}$ in solution.

From the analysis of the bending, the yield stress, or breaking strengh, of $\mathrm{C}_{60} \mathrm{NWs}$ in air was estimated. The yield stress, or breaking strength, $\sigma$ for whiskers can be given by

$$
\sigma=E \frac{r}{\rho}
$$

where $E$ is the Young modulus of whisker, $r$ is the radius of

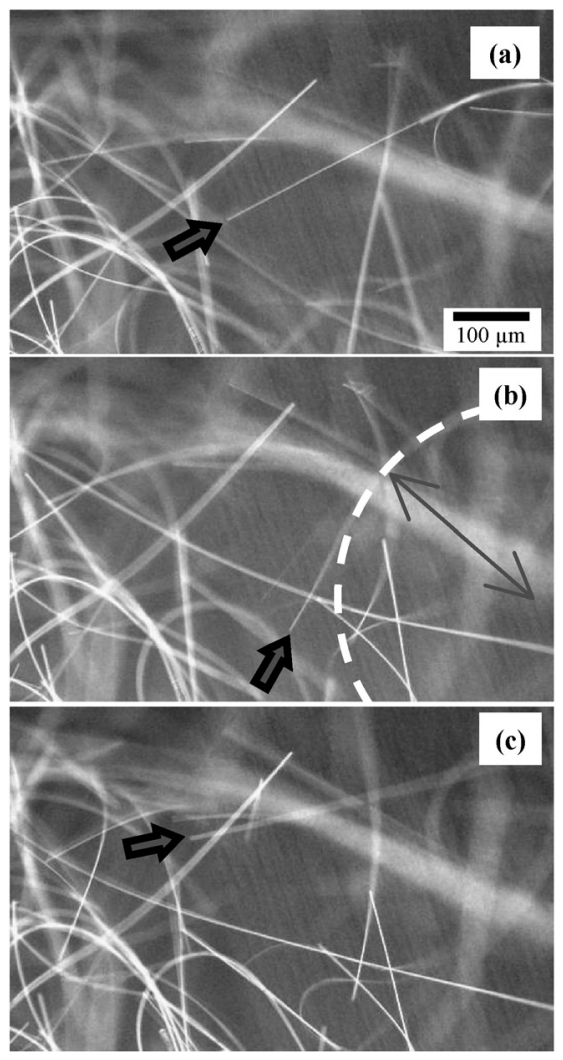

Fig. 1. Successive pictures of the bending deformation of a $\mathrm{C}_{60} \mathrm{NW}$ in solution by wind pressure. The pictures show (a) straight one before applying wind pressure, (b) bended one with the curvature of $290 \mu \mathrm{m}$ by wind pressure, and (c) straight one recovered after freeing from the wind pressure, respectively

whisker, and $\rho$ is the curvature of the bending just before yielding. Eq. (1) was used for $\mathrm{C}_{60} \mathrm{NWs}$ with the diameter of $500 \mathrm{~nm}$ and the curvature of $650 \mu \mathrm{m}$ just before breaking in air. In addition, the Young's modulus of $\mathrm{C}_{60} \mathrm{NWs}$ in air can be assumed to be $20 \mathrm{GPa}$ corresponding to that of $\mathrm{C}_{60}$ bulk crystals, since $\mathrm{C}_{60} \mathrm{NWs}$ in air have fcc structure similar to that of the $\mathrm{C}_{60}$ bulk crystals. Substituting these values into eq. (1), the yield stress, or breaking strength, of $\mathrm{C}_{60} \mathrm{NWs}$ in air was estimated to be $7.7 \mathrm{MPa}$. This value is large by one order compared with $0.7 \mathrm{MPa}$ in $\mathrm{C}_{60}$ bulk crystals as has reported previously. 


\title{
Bending Deformation of $\mathrm{C}_{60}$ Nanowhiskers in Solution and Air
}

\author{
Mami Watanabe* Non-member \\ Kun'ichi Miyazawa** Member \\ Kenichi Kojima* Non-member \\ Masaru Tachibana* Member
}

The mechanical properties of $\mathrm{C}_{60}$ nanowhiskers $\left(\mathrm{C}_{60} \mathrm{NWs}\right)$ grown in a solution by a liquid-liquid interfatical precipitation method were investigated by a bending method. $\mathrm{C}_{60} \mathrm{NWs}$ in the solution exhibited large elastic deformation where they recovered for large bending with a curvature of $290 \mu \mathrm{m}$. On the other hand, $\mathrm{C}_{60} \mathrm{NWs}$ in air were broken even for small bending with only a $650 \mu \mathrm{m}$ curvature. From the analysis of the bending, the yield stress or breaking stress for $\mathrm{C}_{60} \mathrm{NWs}$ in air is estimated to be 7.7 $\mathrm{MPa}$, that is larger by one order compared with $0.7 \mathrm{MPa}$ for bulk $\mathrm{C}_{60}$ crystals. In addition, it is shown that the Young's modulus for $\mathrm{C}_{60} \mathrm{NWs}$ in the solution is $8.9 \mathrm{GPa}$, that is below a half of $20 \mathrm{GPa}$ for bulk $\mathrm{C}_{60}$ crystals.

Keywords : $\mathrm{C}_{60}$ nanowhiskers, mechanical property, raman spectroscopy

\section{Introduction}

Solution-grown $\mathrm{C}_{60}$ crystals have various structures and shapes that depend on organic solvent, growth temperature and so on. Recently, a new type of $\mathrm{C}_{60}$ needle-like crystals called $\mathrm{C}_{60}$ nanowhiskers $\left(\mathrm{C}_{60} \mathrm{NWs}\right)$ were obtained in a solution by $\mathrm{a}$ liquid-liquid interfacial precipitation (LLIP) method, where they nucleate at the interface between a toluene solution of $\mathrm{C}_{60}$ and isopropyl alcohol ${ }^{(1)}$. Typical $\mathrm{C}_{60} \mathrm{NWs}$ are less than $500 \mathrm{~nm}$ in diameter and more than $100 \mu \mathrm{m}$ in length. The high aspect ratio of $\mathrm{C}_{60} \mathrm{NWs}$ with the small diameter is very unique, compared with those of any needle-like $\mathrm{C}_{60}$ crystals reported previously.

From the structural characterization by high resolution transmission electron microscopy, it was found that $\mathrm{C}_{60} \mathrm{NWs}$ have a face-centered cubic ( $\mathrm{fcc}$ ) structure similar to that of intrinsic $\mathrm{C}_{60}$ crystals and their growth direction corresponds to a [110] close-packed direction ${ }^{(1)}$. In addition, Raman scattering measurements have shown that $\mathrm{C}_{60} \mathrm{NWs}$ are condensed with weak van der Waals (vdW) forces and are easily polymerized under laser irradiation ${ }^{(2)}$. Such shapes and structural characteristics of $\mathrm{C}_{60} \mathrm{NWs}$ may provide us with various applications such as nano-scale electronic devices and field emitters.

Some interesting physical properties, e.g. mechanical ${ }^{(3)}$ and electrical properties ${ }^{(4)}$, of $\mathrm{C}_{60} \mathrm{NWs}$ have been also reported. However, most of such previous characterizations of $\mathrm{C}_{60} \mathrm{NWs}$ were carried out for dried specimens in air or a vacuum. $\mathrm{C}_{60}$ molecules should be solvated when they are in the growth solution. More recently, it has been reported that the structure of $\mathrm{C}_{60} \mathrm{NWs}$ in air or a vacuum is different from that of original one grown in the solution $^{(5)} \cdot \mathrm{C}_{60} \mathrm{NWs}$ in the solution have a hexagonal solvated structure and their growth direction corresponds to a [0001] direction. The hexagonal solvated structure rapidly changes into

* International Graduate School of Arts and Sciences, Yokohama City University,

22-2 Seto, Kanazawa-ku, Yokohama 236-0027

** Fullerene Engineering Group, Advanced Nano Materials Laboratory, National Institute for Materials Science

1-1 Namiki, Tsukuba, Ibaraki, 305-0044 the fcc structure by drying, i.e. the evaporation of solvent molecules in air or a vacuum. It is expected that the physical properties of $\mathrm{C}_{60} \mathrm{NWs}$ in the solution are different from those in air or a vacuum. In this paper, we report the bending properties of $\mathrm{C}_{60} \mathrm{NWs}$ in a solution and air.

\section{Experimental Procedure}

$\mathrm{C}_{60} \mathrm{NWs}$ were grown by the LLIP method in below procedure as reported previously ${ }^{(6)}$. As-received $\mathrm{C}_{60}$ powder $(>99.98 \%)$ was used as a source material. The saturated solution of $\mathrm{C}_{60}$ and toluene was prepared and poured into a glass bottle $(13.5 \mathrm{ml})$. Then, the isopropyl alcohol was gently added into the bottle to form a liquid-liquid interface, where the upper phase is isopropyl alcohol and the lower phase is a toluene solution of $\mathrm{C}_{60}$. The bottle was loosely capped and kept under illumination at $294 \mathrm{~K}$. The nucleation of $\mathrm{C}_{60} \mathrm{NWs}$ can mainly occur at the liquid-liquid interface. The interface disappears due to the natural diffusion of the solution within 24 hours. After the disappearance of the interface, the growth rather than the nucleation can mainly occur. $\mathrm{C}_{60} \mathrm{NWs}$ grown for more than one month exceed $1 \mathrm{~mm}$ in length but are still below $500 \mathrm{~nm}$ in diameter. These specimens were used for the measurements.

The LLIP method included some $\mathrm{C}_{60}$ bulk crystals as impurities. They predominantly contribute to X-ray diffraction (XRD) and Raman spectra. To obtain intrinsic XRD and Raman spectra of $\mathrm{C}_{60} \mathrm{NWs}$, as-grown crystals were purified by several filtrations and then highly condensed by the centrifugation. The collective $\mathrm{C}_{60} \mathrm{NWs}$ as shown in Fig. 1 were used in XRD and Raman measurements.

The structure and intermolecular interaction of $\mathrm{C}_{60} \mathrm{NWs}$ were examined by XRD (RINT X-ray Diffractometer, Rigaku) and Raman spectroscopy (IFS 66v/S, FRA 106/S, Bruker). To confirm these features in the growth solution and air, the time evolution of the XRD and Raman spectra of $\mathrm{C}_{60} \mathrm{NWs}$ during the drying was measured. In the measurements, the specimens were mounted on a glass sample holder immediately after the sampling from the growth solution. XRD patterns were obtained with a $0.1542 \mathrm{~nm}$ of $\mathrm{Cu} \mathrm{K} \alpha$. The Raman spectra were taken with a $1064 \mathrm{~nm}$ excitation 


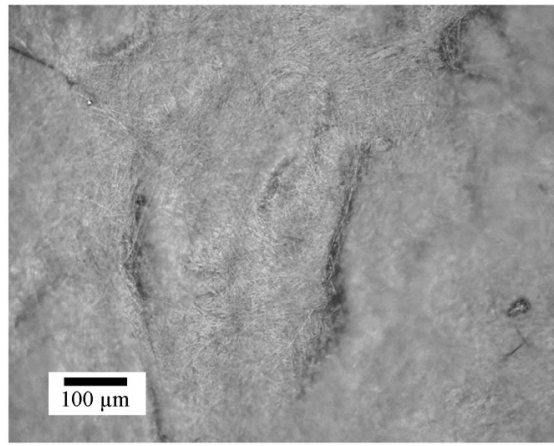

Fig. 1. Collective $\mathrm{C}_{60}$ nanowhiskers $\left(\mathrm{C}_{60} \mathrm{NWs}\right)$, purified by several filtrations and then condensed by the centrifugation. Note that it is difficult to observe $\mathrm{C}_{60} \mathrm{NWs}$ individually since they are highly condensed.

from a YAG laser in order to avoid the photo-polymerization of $\mathrm{C}_{60}$ by excitation.

The mechnaical properties of $\mathrm{C}_{60} \mathrm{NWs}$ in the solution and air were investigated by a bending method. To observe the bending behavior in the solution, the specimens were mounted on the glass plate with droplets, from the growth solution. The bending deformation for both specimens in the solution and air was carried out by applying a wind pressure with a fan. The wind pressure or the stress applied to $\mathrm{C}_{60} \mathrm{NWs}$ could not be measured in this experiment. The observation of the bending behavior was carried out in-situ by using an optical microscope (BX51, Olympus) with a charge coupled device (CCD).

\section{Result and Discussion}

Fig. 2 shows the time evolution of XRD pattern of $\mathrm{C}_{60} \mathrm{NWs}$ during the drying process. The XRD pattern taken for $25 \mathrm{~min}$ after the sampling from the glass bottles showed sharp peaks. The sharp peaks were observed for up to $230 \mathrm{~min}$ whereas the broad peaks were at the similar positions as those for the intrinsic $\mathrm{C}_{60}$ bulk crystals and indexed by the fcc system with a cell dimension of $a=1.44 \mathrm{~nm}$. This indicates that the structure was changed into the fcc, from a solvated structure by the evaporation of solvent molecules in air. The pattern taken for 25 min in Fig. 2 were indexed by the hexagonal system with cell dimensions $a=2.43 \mathrm{~nm}$ and $c=1.01 \mathrm{~nm}(a / c=2.41)$. Such a change in structure by drying is similar to that as has been reported previously.

The time evolution of the Raman spectrum of $\mathrm{C}_{60} \mathrm{NWs}$ during the drying was also measured to examine the intermolecular interaction in $\mathrm{C}_{60} \mathrm{NWs}$ in the solution and air. Fig. 3 shows the time evolution of main Raman bands at 270, 495, and $1468 \mathrm{~cm}^{-1}$ in air, which can be associated with $\mathrm{H}_{\mathrm{g}}(1)$ squashing, $\mathrm{A}_{\mathrm{g}}(1)$ breathing, $\mathrm{A}_{\mathrm{g}}(2)$ pentagonal pinch modes of $\mathrm{C}_{60}$ molecules, respectively. For the comparison, Raman bands in $\mathrm{C}_{60}$ molecules in a toluene solution, the hexagonal $\mathrm{C}_{60}$ bulk crystals grown from a toluene solution, and fcc $\mathrm{C}_{60}$ bulk crystals grown from sublimation are included in Fig. 3.

No significant change in the peak profiles of $A_{g}(1)$ and $A_{g}(2)$ modes is observed although the frequencies of the peaks are shifted. On the other hand, the peak profile of $\mathrm{H}_{\mathrm{g}}(1)$ for $\mathrm{C}_{60}$ molecules is quite different from that for the crystalline $\mathrm{C}_{60}$. Namely, the peak for $\mathrm{C}_{60}$ molecules was fitted by a Lorentzian curve while that for the crystals can be fitted by several curves. Such splitting in $\mathrm{H}_{\mathrm{g}}(1)$ mode for the crystals should be attributed to crystal field due to regular molecular arrangement in the

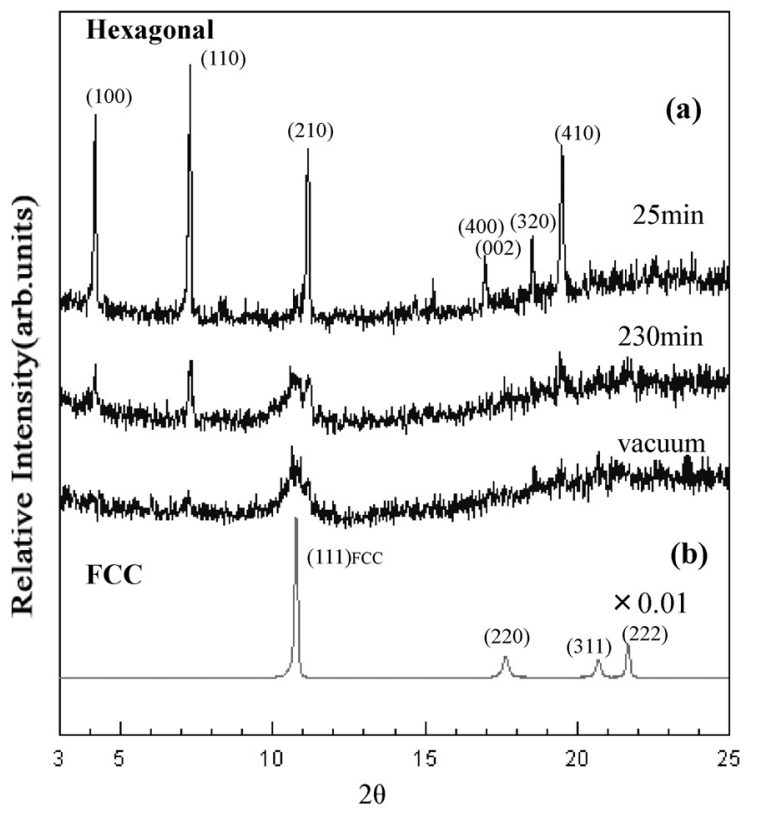

Fig. 2. Time evolution of XRD pattern for $\mathrm{C}_{60} \mathrm{NWs}$ during the drying process (a). The XRD pattern for intrinsic $\mathrm{C}_{60}$ bulk crystals with the fcc structure grown by sublimation (b) is included in the figure. The XRD patterns were obtained with $0.1542 \mathrm{~nm}$ of $\mathrm{Cu} \mathrm{K \alpha}$.

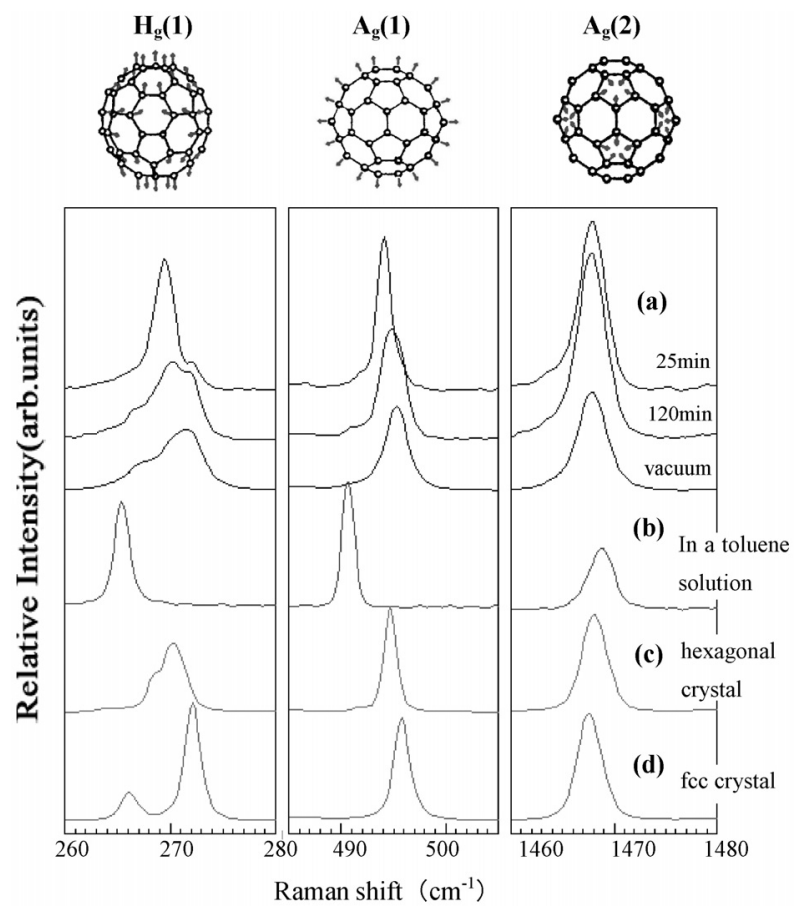

Fig. 3. Time evolution of main Raman bands for $\mathrm{C}_{60} \mathrm{NWs}$ during the drying process (a). The Raman bands at 270,495 , and $1468 \mathrm{~cm}^{-1}$ can be associated with $H_{\mathrm{g}}(1)$ "squashing", $A_{\mathrm{g}}(1)$ "breathing", $A_{\mathrm{g}}(2)$ "pentagonal pinch" modes of $\mathrm{C}_{60}$ molecules, respectively. For the comparison, Raman bands in $\mathrm{C}_{60}$ molecules in a toluene solution (b), the hexagonal $\mathrm{C}_{60}$ bulk crystals grown from a toluene solution (c), and the fcc $\mathrm{C}_{60}$ bulk crystals grown from sublimation (d) are include in the figure. The Raman spectra were taken with $1064 \mathrm{~nm}$ excitation. 


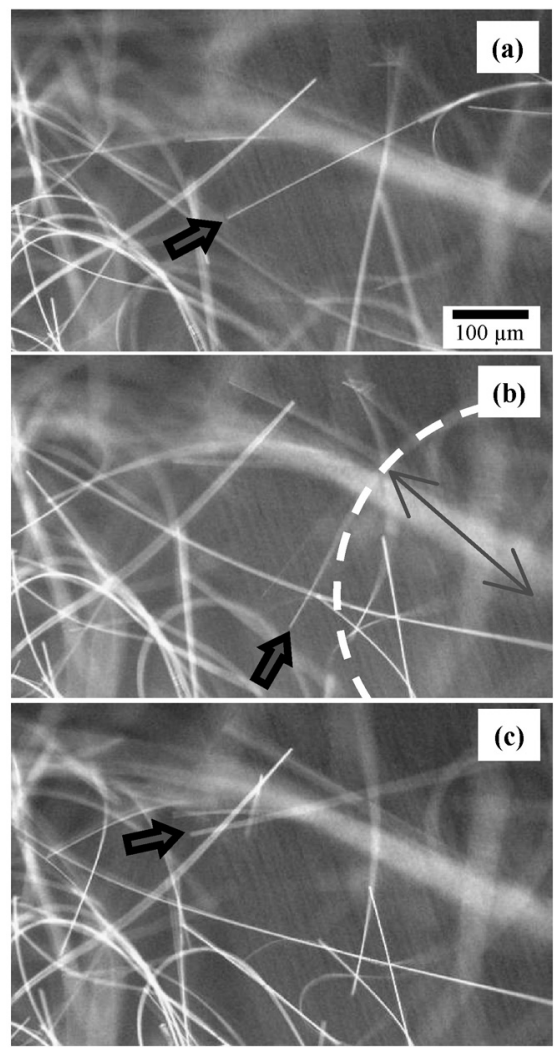

Fig. 4. Successive pictures of the bending deformation of a $\mathrm{C}_{60} \mathrm{NW}$ in the solution by a wind pressure. The pictures show (a) straight one before applying a wind pressure, (b) bent one with a curvature of $290 \mu \mathrm{m}$ by a wind pressure, and (c) straight one recovered after freeing from a wind pressure, respectively.

crystals. $\mathrm{C}_{60} \mathrm{NWs}$ in the solution have the hexagonal structure which is similar to that of $\mathrm{C}_{60}$ bulk crystals grown from a toluene solution. However, no clear splitting in $\mathrm{H}_{\mathrm{g}}(1)$ peak was observed for $\mathrm{C}_{60} \mathrm{NWs}$ in the solution. This means that $\mathrm{C}_{60} \mathrm{NWs}$ in the solution have a smaller crystal field, i.e. smaller intermolecular interaction although they have a regular molecular arrangement, similar to that of $\mathrm{C}_{60}$ bulk crystals. It is expected that such intermolecular interaction in $\mathrm{C}_{60} \mathrm{NWs}$ with solvated strucutre can lead to unique physical properties differing from those in air.

For such $\mathrm{C}_{60} \mathrm{NWs}$ in a solution and air, the bending deformation was investigated. Fig. 4 shows the successive pictures of the bending behavior of a $\mathrm{C}_{60} \mathrm{NW}$ which has $500 \mathrm{~nm}$ in diameter and more than $100 \mu \mathrm{m}$ in length. The straight $\mathrm{C}_{60} \mathrm{NW}$ before applying a wind pressure is indicated by the arrow in Fig. 4(a). The $\mathrm{C}_{60} \mathrm{NW}$ was bent with a wind pressure and reached a curvature of $290 \mu \mathrm{m}$, as shown in Fig. 4(b). Freeing from a wind pressure, the $\mathrm{C}_{60} \mathrm{NW}$ recovered to original straight shape, as shown in Fig. 4(c). Such behavior of the bending shows that $\mathrm{C}_{60} \mathrm{NWs}$ in the solution exhibit large elastic deformation.

The bending experiments were also carried out for $\mathrm{C}_{60} \mathrm{NWs}$ in air, as shown in Fig. 5. A straight $\mathrm{C}_{60} \mathrm{NW}$ before applying a wind pressure is indicated by the arrow in Fig. 5(a). The $\mathrm{C}_{60} \mathrm{NW}$ was bent under a wind pressure, as shown in Fig. 5(b). However, the $\mathrm{C}_{60} \mathrm{NW}$ in air was broken just after it was bent with a curvature of only $650 \mu \mathrm{m}$, as shown in Fig. 5(b). Therefore, $\mathrm{C}_{60} \mathrm{NWs}$ in air exhibit smaller elastic deformation, i.e. are relatively brittle, compared with that in the solution. As clearly shown, $\mathrm{C}_{60} \mathrm{NWs}$ in

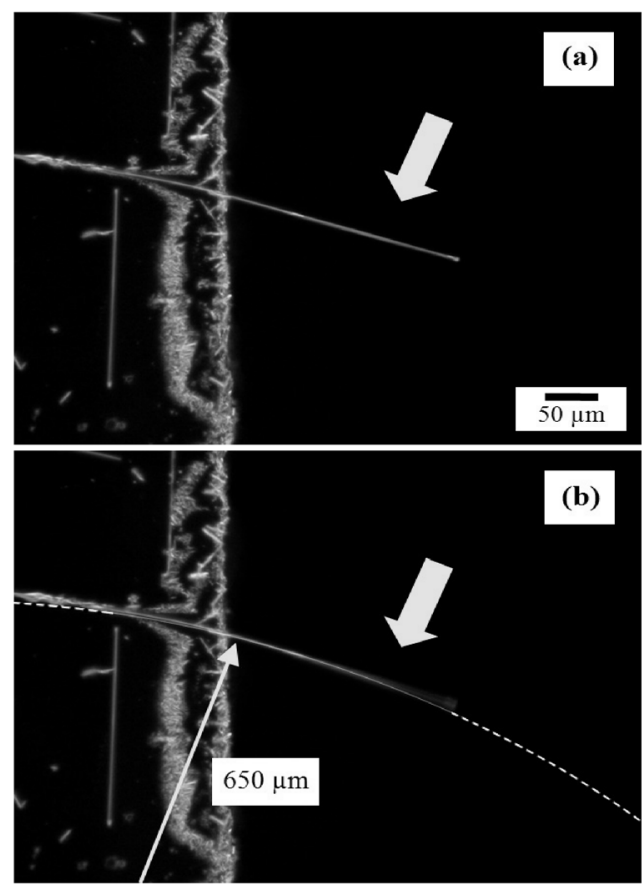

Fig. 5. Successive pictures of the bending deformation of a $\mathrm{C}_{60} \mathrm{NW}$ in air. The stress was applied by a wind pressure. The pictures show (a) straight one before applying a wind pressure, and (b) bent one with the curvature of $650 \mu \mathrm{m}$ by a wind pressure, respectively. The further bending leads to the break of the $\mathrm{C}_{60} \mathrm{NW}$.

the solution show large elastic deformation. This might be due to the solvated structure of $\mathrm{C}_{60} \mathrm{NWs}$ with weak intermolecular interaction.

The yield stress or breaking strength for whiskers can be estimated from loop-like deformation ${ }^{(7)}$. In the deformation, the curvature of the loop increases with stress, and further increases in stress leads to a yield or break of the whisker. The yield stress $\sigma$ of the whisker can be estimated from the curvature just before yielding, and is given by

$$
\sigma=E \frac{r}{\rho}
$$

where $E$ is the Young's modulus of whisker, $r$ is the radius of whisker, and $\rho$ is the curvature of the loop just before yielding ${ }^{(7)}$. Eq. (1) can be obtained from the theory on beam deformation with $\mathrm{r} \ll \rho$. Therefore, Eq. (1) is available for $\mathrm{C}_{60} \mathrm{NWs}$ with the diameter of $500 \mathrm{~nm}$ and the curvature of $650 \mu \mathrm{m}$ just before breaking in air in Fig. 5(b). In addition, the Young's modulus of $\mathrm{C}_{60} \mathrm{NWs}$ in air can be assumed to be $20 \mathrm{GPa}$ corresponding to that of $\mathrm{C}_{60}$ bulk crystals ${ }^{(8)}$, since $\mathrm{C}_{60} \mathrm{NWs}$ in air have the fcc structure condensed with weak vdW interaction similar to that in $\mathrm{C}_{60}$ bulk crystals as mentioned above. Substituting these values into Eq. (1), the yield stress or breaking strength for $\mathrm{C}_{60} \mathrm{NWs}$ in air is estimated to be $7.7 \mathrm{MPa}$. This value is larger by one order compared with $0.7 \mathrm{MPa}$ in $\mathrm{C}_{60}$ bulk crystals as has been reported previously ${ }^{(9)}$.

Assuming that the yield stress $\left(7.7 \mathrm{MPa}\right.$ ) for $\mathrm{C}_{60} \mathrm{NW}$ in air as mentioned above is similar to the stress applied to $\mathrm{C}_{60} \mathrm{NW}$ in the solution in Fig. 4, a Young's modulus of $\mathrm{C}_{60} \mathrm{NWs}$ in the solution can be also estimated by using Eq. (1). For the $\mathrm{C}_{60} \mathrm{NW}$ with the diameter of $500 \mathrm{~nm}$ and the curvature of $290 \mu \mathrm{m}$ in the solution in Fig. 4(b), the Young's modulus is estimated to be $8.9 \mathrm{GPa}$, that is 
below a half of $20 \mathrm{GPa}$ for $\mathrm{C}_{60}$ bulk crystals. In this experiment, it is difficult to obtain more reliable values since the stress applied to $\mathrm{C}_{60} \mathrm{NWs}$ could not be measured. However, these values obtained in this paper are very important as reference data for further study of mechanical properties of $\mathrm{C}_{60} \mathrm{NWs}$ and the applications.

\section{Conclusion}

We have shown in-situ observation of the bending deformation of $\mathrm{C}_{60} \mathrm{NWs}$ in not only air but also solution. It was shown that $\mathrm{C}_{60} \mathrm{NWs}$ in the solution exhibit large elastic deformation compared with that in air. Such elastic property of $\mathrm{C}_{60} \mathrm{NWs}$ with solvated structure might lead to new applications.

\section{Acknowledgement}

This work was supported by a Strategic Reseach Project (K19044) in Yokohama City University.

(Manuscript received Nov. 28, 2007, revised Apr. 1, 2008)

\section{References}

(1) K. Miyazawa, Y. Kuwasaki, A. Obayashi, and M. Kuwabara : " $\mathrm{C}_{60}$ nanowhiskers formed by the liquid-liquid interfacial precipitation method", J. Mater. Res., Vol.17, pp.83-88 (2002)

(2) M. Tachibana, K. Kobayashi, T. Uchida, K. Kojima, K. Tanimura, and K. Miyazawa : "Photo-assisted growth and polymerization of $\mathrm{C}_{60}$ nanowhiskers", Chem. Phys. Lett., Vol.374, pp.279-285 (2003)

(3) K. Asaka, R. Kato, K. Miyazawa, and T. Kizuka : "Buckling of $\mathrm{C}_{60}$ whiskers", Appl. Phys. Lett., Vol.89, pp.071912-1-071912-3 (2006)

(4) K. Ogawa, T. Kato, A. Ikegami, H. Tsuji, N. Aoki, and Y. Ochiai : "Electrical properties of field-effect transistors based on $\mathrm{C}_{60}$ nanowhiskers", J.P. Bird, Appl. Phys. Lett., Vol.88, pp.112109-1- 112109-3 (2006)

(5) J. Minato and K. Miyazawa : Carbon, "Solvated structure of $\mathrm{C}_{60}$ nanowhiskers", Vol.43, pp.2837-2841 (2005)

(6) K. Kobayashi, M. Tachibana, and K. Kojima : "Photo-assisted growth of $\mathrm{C}_{60}$ nanowhiskers from solution”, J. Cryst. Growth, Vol.274, pp.617 (2005)

(7) T. Kaneko : "Whiskers", Kyouritsu Shuppan, Tokyo (1993)

(8) M. S. Dresselhaus, G. Dresselhaus, and P. C. Eklund : "Science of Fullerenes and Carbon Nanotubes", Academic Press, New York (1996)

(9) T. Komatsu, M. Tachibana, and K. Kojima : Plastic deformation of $\mathrm{C}_{60}$ single crystals", Phil Mag., Vol.81, pp.659-666 (2001)

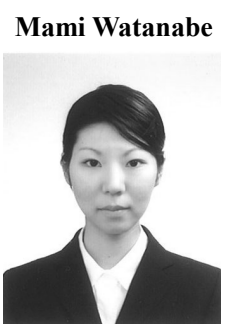

(Non-member) was born in 1985. She received B.S. in 2007 from Yokohama City University. She is now a graduate student of master couse of Graduate School of Yokohama City University (Tachibana Lab)

Kun'ichi Miyazawa (Member) was born in 1955. He received D.E.

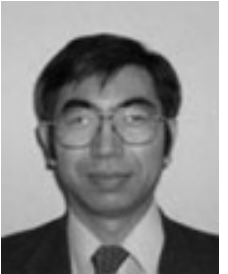
degree from The University of Tokyo in 1987. He was a lecturer of School of Engineering, The University of Tokyo from 1989 to 2002, and moved to National Institute for Materials Science in 2002. He is now engaged in the synthesis, characterization and application of low-dimensional fullerene materials such as fullerene nanowhiskers, fullerene nanotubes and fullerene nanosheets.

Kenichi Kojima

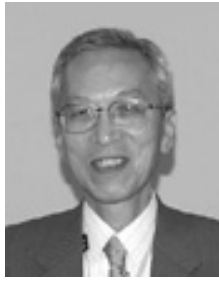

Masaru Tachibana

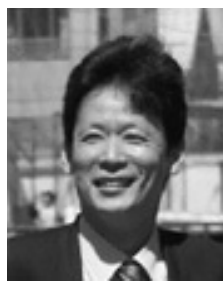

(Non-member) was born in 1942 . He received D.E. degree from Tohoku University in 1971. He is now a professor of Yokohama City University.

nanotubes and fullerenes.

(Member) was born in 1963. He received D.E. degree from Waseda University in 1995. He is now a professor of Yokohama City University. His research interests are the physical properties of various kinds of molecular crystals composed of small molecules to giant ones such as proteins. Current research projects involve the development of synthesis and crystal growth of novel materials such as carbon 